\title{
A role for connexin-43 in Duchenne muscular dystrophy cardiomyopathy
}

\author{
Robin M. Shaw ${ }^{1}$ and Jeffrey E. Saffitz ${ }^{2}$ \\ ${ }^{1}$ Nora Eccles Harrison Cardiovascular Research and Training Institute, University of Utah, Salt Lake City, Utah, USA. ²Department of Pathology, Beth Israel Deaconess Medical Center, Boston, Massachusetts, USA.
}

\begin{abstract}
The cardiomyopathy of Duchenne muscular dystrophy (DMD) is an important cause of morbidity and mortality in affected males with this dreaded muscle disease. Previous studies have implicated changes in expression and subcellular localization of connexin-43 (Cx43), the major ventricular gap junction protein, in DMD cardiomyopathy. In this issue of the $J C I$, Himelman et al. explore how hypophosphorylation of Cx43 at a triplet of serine residues (S325/S328/S330) in the regulatory C-terminus contributes to multiple features of the cardiomyopathy phenotype. Using a mouse model of DMD cardiomyopathy in which phosphomimetic glutamic acids are substituted for serines at these residues in Cx43, Himelman et al. observed reduced gap junction remodeling and lateralization of Cx43 immunosignals, protection against isoproterenol-induced arrhythmias, and improved $\mathrm{Ca}^{2+}$ homeostasis. This study contributes to the understanding of pathologic Cx43 remodeling and encourages further research into developing strategic interventions to mitigate cardiac dysfunction and arrhythmias in DMD patients.
\end{abstract}

\section{The cardiomyopathy of} Duchenne muscular dystrophy Duchenne muscular dystrophy (DMD) is a devastating $\mathrm{X}$-linked disease caused by absence of the giant muscle-specific protein dystrophin. It leads to progressive skeletal muscle weakness culminating in respiratory dysfunction and fatal complications. Life expectancy is generally less than 30 years, but improved clinical management has allowed most patients to survive beyond adolescence, by which time the cardiomyopathy of DMD manifests in many.

As in all the familial, nonischemic cardiomyopathies, the mechanisms by which monogenic loss-of-function variants produce the associated clinical and pathological cardiac disease phenotypes are highly complex. Corresponding specific mutations exert direct and indirect effects and involve multiple molecular pathways.
This is certainly true for DMD cardiomyopathy. Much of what we know about this condition comes from studies in $\mathrm{mdx}$ mice, a widely used model of DMD involving a spontaneous point mutation in $\mathrm{Dmd}$, which causes muscle cells to produce a small, nonfunctional dystrophin protein (1). As reviewed by Shirokova and Niggli (2), cardiac myocytes from mdx mice show many abnormalities, including increased fragility of the sarcolemma, increased transmembrane ionic fluxes, elevated resting cytosolic levels of $\mathrm{Na}^{+}$and $\mathrm{Ca}^{2+}$, increased intracellular $\mathrm{Ca}^{2+}$ responses to mechanical stimulation, hypersensitive excitation-contraction (EC) coupling, and a shift in intracellular and mitochondrial redox state toward a more oxidative state. To what extent these complex abnormalities are directly linked to loss of dystrophin is unknown. Increased sarcolemmal
Related Article: p. 1713

Conflict of interest: The authors have declared that no conflict of interest exists.

Copyright: @ 2020, American Society for Clinical Investigation.

Reference information: / Clin Invest. 2020;130(4):1608-1610. https://doi.org/10.1172/JCI135007.

fragility is presumably a direct result of loss of dystrophin, whereas other defects including abnormal ionic homeostasis and shifts toward a heightened oxidative state are likely downstream effects of multiple, complex disease pathways.

\section{Connexin remodeling in DMD cardiomyopathy}

Abnormalities in connexin-43 ( $\mathrm{Cx} 43)$, the major ventricular gap junction protein, have been identified in DMD cardiomyopathy. For example, increased $\mathrm{Cx} 43$ protein content and redistribution of $\mathrm{Cx} 43$ from gap junctions concentrated at intercalated disks to sites subjacent to the lateral sarcolemma, so-called lateralization, have been described in myocardium from DMD patients and mdx mice (3). This is accompanied by changes in $\mathrm{Cx} 43$ phosphorylation suggesting alterations in gap junctional regulation. But again, whether these changes are a cause or an effect of the complex disease pathways in DMD cardiomyopathy can be difficult to determine.

In this issue of the JCI, Himelman et al. (4) provide evidence that dysregulation of $\mathrm{Cx} 43$ does, in fact, contribute to the pathogenesis of DMD cardiomyopathy, but exactly how this works remains unclear. The authors first identified reduced phosphorylation of Cx43 at serines S325/S328/ $\mathrm{S} 330$ in the hearts of $\mathrm{mdx}$ mice. These serine residues reside in the $\mathrm{C}$-terminal intracellular tail of the Cx43 molecule. Phosphorylation and dephosphorylation of these and other $\mathrm{C}$-terminal residues under basal conditions and in response to injury regulate protein-protein interactions involved in Cx43 trafficking, turnover, and function (5). These are extraordinarily complex mechanisms, and our understanding of such regulation in health and disease is still far from complete. Nevertheless, recognizing that hypophosphorylation of $\mathrm{Cx} 43$ at serines S325/S328/S330 could be related to changes in Cx43 expression, distribution, and function in DMD cardiomyopathy, Himelman et al. (4) then crossed mdx mice with a 
line originally created by Glenn Fishman in which the $\mathrm{Cx} 43$ serine triplet was replaced with either phosphomimetic glutamic acids or nonphosphorylatable alanines (6). Himelman and colleagues found that various features of the DMD cardiomyopathy phenotype were mitigated in mdx mice with germline knockin of glutamic acids at S325/ S328/S330 (referred to as mdxS3E), but not in those with alanines (mdxS3A) (4). For example, when subjected to hypo-osmotic shock, a method developed by Ernst Niggli (2), isolated mdx cardiac myocytes exhibited larger $\mathrm{Ca}^{2+}$ signals compared with WT myocytes. Notably, these $\mathrm{Ca}^{2+}$ signals were reduced in mdxS3E myocytes. Further, the $\beta-1$ and $\beta-2$ adrenergic receptor agonist, isoproterenol, induced more arrhythmias in mdx mice than in WT mice, and these too were reduced in $\mathrm{mdxS} 3 \mathrm{E}$ mice. Gap junction remodeling was also reduced in the hearts of mdxS3E mice. The amount of lateralized Cx43 immunoreactive signal was decreased and more signal appeared at intercalated disks, a finding corroborated by independent biochemical assays. In related previous work (7), these same authors showed that pretreating mdx mice with Gap19, a 9-amino acid peptide from the intracellular cytoplasmic loop domain of human $\mathrm{Cx} 43$, limited arrhythmias induced by isoproterenol. Gap19 is thought to block the actions of $\mathrm{Cx} 43$ hemichannels, which, in turn, are enriched within extrajunctional sites. In the work reported here, Himelman et al. perfused hearts with the membrane-impermeant dye ethidium bromide, which can enter cells via open hemichannels, but not through gap junctions. The researchers observed marked uptake in ventricular myocytes in both control $\mathrm{mdx}$ and mdxS3A hearts compared with WT hearts and mdxS3E hearts, consistent with reduced levels of open $\mathrm{Cx} 43$ hemichannels in mdxS3E myocytes (4).

\section{Mechanistic insights}

Taken together, these findings clearly demonstrate that $\mathrm{Cx} 43$ remodeling is mitigated in $\mathrm{mdxS} 3 \mathrm{E}$ mice, as shown by preservation of $\mathrm{Cx} 43$ in gap junctions at intercalated disks, reduced lateral redistribution of $\mathrm{Cx} 43$, and reduced hemichannels activity. To gain insights into the mechanisms responsible for reduced $\mathrm{Cx} 43$ remodeling, Himelman et al. focused attention on $\mathrm{NAD}(\mathrm{P}) \mathrm{H}$ oxidase isoform2 (NOX2), shown previously as a major source of reactive oxygen species in mdx mice $(2,8)$. They observed reduced amounts of NOX2 in mdxS3E hearts compared with control mdxhearts. Again, turning to previous work linking aberrant activity of oxidized $\mathrm{Ca}^{2+}$ calmodulin-dependent protein kinase II (CamKII) (perhaps mediated at least in part by NOX2) to abnormal $\mathrm{Ca}^{2+}$ homeostasis and arrhythmias in mdx mice (9), Himelman et al. measured total $\mathrm{Cx} 43$ and phosphoserine-Cx43 (pS-Cx43) in lysates from $\mathrm{mdx}$ mice and a previously described line (mdx:MM-VV) in which the CaMKII regulatory domain is protected from oxidation. They observed an increase in the proportion of $\mathrm{pS}-\mathrm{Cx} 43$ along with biochemical evidence of increased junctional Cx43 in mdx:MM-VV hearts compared with mdx hearts. Given that CaMKII is capable of phosphorylating $\mathrm{Cx} 43$ at serines S325/S328/S330, Himelman et al. thus build a reasonably compelling case suggesting that oxidation of CaMKII, perhaps by NOX2 and/or other agents, contributes to reduced $\mathrm{Cx} 43$ phosphorylation at serines S325/S328/S330, which in turn, is linked to $\mathrm{Cx} 43$ remodeling and associated changes of abnormal $\mathrm{Ca}^{2+}$ homeostasis in DMD cardiomyopathy (4).

Of course, among the most important clinical features of DMD cardiomyopathy are contractile dysfunction and myocardial damage, and here is where the limitations in $\mathrm{mdxS} 3 \mathrm{E}$ mice are revealed. While there was some improvement in contractile performance (percentage of ejection fraction) and a reduction in myocardial fibrosis in older (14 to 18 months) mdxS3E mice compared with age-matched $\mathrm{mdx}$ mice, these salutary effects were relatively modest. In the end, dystrophin was still absent in mdxS3E mice and the mice still suffered from cardiomyopathy.

A fundamental test of whether DMDassociated hypophosphorylation of $\mathrm{Cx} 43$ at $\mathrm{S} 325 / \mathrm{S} 328 / \mathrm{S} 330$ is causative in DMD cardiomyopathy is to explore whether hypophophorylation of $\mathrm{Cx} 43$ alone is sufficient to result in cardiomyopathy. Phosphomimetic S3A substitutions in $\mathrm{Cx} 43$ provide an opportunity to test for such sufficiency. In the original report from Fishman's group (6), S3A substitutions failed to result in hypertrophy, fibrosis, or diminished contractile function, nor did they lead to decreased survival. Thus, S3A substitutions in $\mathrm{Cx} 43$ per se are not detrimental to cardiac function. Furthermore, as reported by Himelman et al., S3A substitutions in an $\mathrm{mdx}$ background ( $\mathrm{mdxS} 3 \mathrm{~A}$ ) are surprisingly protective against cardiac fibrosis and actually improve survival after isoproterenol challenge (4). This is contrary to what would be expected from maladaptive hypophosphorylation. Thus, while studies from both Fishman's group (6) and Himelman et al. (4) support a role for $\mathrm{S} 3 \mathrm{E}$ in protecting against $\mathrm{Cx} 43$ remodeling and arrhythmogenic death, the conclusion that S3E substitutions protect against heart dysfunction and, by extension, that S325/S328/S330 hypophosphorylation causes mdx cardiomyopathy, is less compelling.

\section{New directions}

Nevertheless, the findings of Himelman et al. are of interest and speak to complex relationships among $\mathrm{Cx} 43$ phosphorylation, distribution, trafficking, and function. Clearly, substituting serines with phosphomimetic glutamic acids at S325/ S328/S330 in Cx43 delays Cx43 remodeling in DMD cardiomyopathy (4). However, such Cx43 remodeling (increased lateralization, more hemichannels, less $\mathrm{Cx} 43$ in gap junctions at intercalated disks) occurs in many disease settings. For example, it is seen in infarct border zones, in heart failure, and in systemic and pulmonary hypertension (10). Indeed, Cx43 remodeling may be regarded as a default response to injury in cardiac myocytes. Thus, the pathogenesis of DMD cardiomyopathy may involve a combination of injurious mechanisms, an initial inciting injury mediated by direct effects of lack of dystrophin, and additional, subsequent mechanisms mediated by $\mathrm{Cx} 43$ remodeling.

The results of Himelman et al. highlight the complex bidirectional relationships among $\mathrm{Cx} 43$ channel function, $\mathrm{Ca}^{2+}$ homeostasis, redox state, and, perhaps, mitochondrial function (4). Indeed, Cx43 has been shown to localize in mitochondria, and it appears to protect them from ischemic injury (11). In this regard, noncanonical roles of $\mathrm{Cx} 43$ may associate with endogenous truncated isoforms produced by internal translation. The truncated isoform of Cx43, GJA1-20k, occurs in cardiac muscle (12), and its expression is increased with ischemic stress and in 
the failing heart (13). GJA1-20k appears to target mitochondria (14), where it beneficially affects mitochondrial dynamics (14), reduces ROS generation (13), mimics ischemic preconditioning protection of left ventricular function, and protects against ischemic injury (13). The responsible mechanisms appear related to GJA120k interactions with the actin cytoskeleton, affecting both actin and microtubule organization $(14,15)$, not unlike the protective effects observed by Himelman et al. in response to modulation of microtubule polymerization (4). The possibility that S3E phosphomimetic substitutions in $\mathrm{Cx} 43$ potentiate both full-length $\mathrm{Cx} 43$ trafficking as well as the noncanonical roles of GJA1-20k (which originates from the same gene and mRNA product) would support a unifying, though as yet unproven, hypothesis. In this context, improved Cx43 trafficking might serve as a readout for improved metabolic actions of the smaller Cx43 isoform GJA1-20k.

In conclusion, Himelman et al. add to previous studies implicating $\mathrm{Cx} 43$ remodeling in the pathogenesis of $\mathrm{DMD}$ cardiomyopathy (4). Their results also complement other prior studies showing that preservation of Cx43-pS325/328/330 has salutary effects in mouse models of pressure overload hypertrophy. But exactly how this occurs is still not entirely clear. The extent to which preserved gap junctional function contributed to cardi- ac protection is also unclear, but perhaps important. Finally, it is uncertain whether targeting Cx43 remodeling would clinically benefit patients with DMD cardiomyopathy. Studies using AAV to deliver CRISPR/Cas9 gene-editing machinery to remove mutated exons in the dystrophin gene in skeletal and cardiac muscle cells are encouraging (16). Still, the cardiomyopathy of DMD contributes importantly to morbidity and mortality, and insight into how to mitigate its effects is welcomed.

Address correspondence to: Jeffrey E. Saffitz, Department of Pathology, Beth Israel Deaconess Medical Center, 330 Brookline Avenue, Boston, Massachusetts 02215, USA. Phone: 617.667.4343; Email: jsaffitz@ bidmc.harvard.edu.

1. Bulfield G, Siller WG, Wight PA, Moore KJ. $\mathrm{X}$ chromosome-linked muscular dystrophy (mdx) in the mouse. Proc Natl Acad Sci USA. 1984;81(4):1189-1192.

2. Shirokova N, Niggli E. Cardiac phenotype of Duchenne Muscular Dystrophy: insights from cellular studies. J Mol Cell Cardiol. 2013;58:217-224.

3. Gonzalez JP, et al. Normalization of connexin 43 protein levels prevents cellular and functional signs of dystrophic cardiomyopathy in mice. Neuromuscul Disord. 2018;28(4):361-372.

4. Himelman E, et al. Prevention of connexin-43 remodeling protects against Duchenne muscular dystrophy cardiomyopathy. J Clin Invest. 2020;130(4):1713-1727.

5. Solan JL, Lampe PD. Spatio-temporal regulation of connexin 43 phosphorylation and gap junction dynamics. Biochim Biophys Acta Biomembr.
2018;1860(1):83-90.

6. Remo BF, et al. Phosphatase-resistant gap junctions inhibit pathological remodeling and prevent arrhythmias. Circ Res. 2011;108(12):1459-1466.

7. Gonzalez JP, Ramachandran J, Xie LH, Contreras JE, Fraidenraich D. Selective connexin 43 inhibition prevents isoproterenol-induced arrhythmias and lethality in muscular dystrophy mice. Sci Rep. 2015;5:13490.

8. Williams IA, Allen DG. The role of reactive oxygen species in the hearts of dystrophin-deficient mdx mice. Am J Physiol Heart Circ Physiol. 2007;293(3):H1969-H1977.

9. Wang Q, et al. Oxidized CaMKII $\left(\mathrm{Ca}^{2+} /\right.$ calmodulin-dependent protein kinase II) is essential for ventricular arrhythmia in a mouse model of Duchenne muscular dystrophy. Circ Arrhythm Electrophysiol. 2018;11(4):e005682.

10. Delmar M, Laird DW, Naus CC, Nielsen MS, Verselis VK, White TW. Connexins and disease. Cold Spring Harb Perspect Biol. 2018;10(9):a029348.

11. Boengler K, Schulz R. Connexin 43 and mitochondria in cardiovascular health and disease. Adv Exp Med Biol. 2017;982:227-246.

12. Smyth JW, Shaw RM. Autoregulation of connexin43 gap junction formation by internally translated isoforms. Cell Rep. 2013;5(3):611-618.

13. Basheer WA, et al. Stress response protein GJA1-20k promotes mitochondrial biogenesis, metabolic quiescence, and cardioprotection against ischemia/reperfusion injury. JCI Insight. 2018;3(20):121900.

14. Fu Y, et al. Cx43 Isoform GJA1-20k promotes microtubule dependent mitochondrial transport. Front Physiol. 2017;8:905.

15. Basheer WA, et al. GJA1-20k arranges actin to guide $\mathrm{Cx} 43$ delivery to cardiac intercalated discs. Circ Res. 2017;121(9):1069-1080.

16. Min YL, Bassel-Duby R, Olson EN. CRISPR correction of Duchenne muscular dystrophy. Annu Rev Med. 2019;70:239-255. 